\title{
Proceso Y PRODUCTOS DE UNA INTERVENCIÓN PSICOLÓGICA VOCACIONAL ${ }^{\star}$
}

\author{
Liliana da Costa Faria $\star \star$

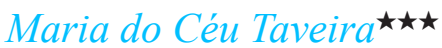

\begin{abstract}
RESUMEN
Este estudio analiza el proceso y los productos de una intervención psicológica vocacional. Participaron 321 estudiantes (178 grupo experimental y 143 grupo control), entre 13 y 17 años. Se evaluó el proceso de intervención a través del Sistema de Reacciones de los Clientes, y los productos a través de las Escala de Exploración Vocacional y de Indecisión Vocacional. El análisis de las sesiones de intervención demuestra que a través de todo el proceso, las reacciones confianza, bienestar, auto-concepto, claridad, responsabilidad, alteración de perspectivas, educación, cambio y falta de reacción, registran cambios estadísticamente significativos, a favor de la intervención. El grupo experimental obtuvo puntaciones estadísticamente significativas superiores en varias escalas de la exploración vocacional y puntaciones estadísticamente significativas inferiores en la indecisión vocacional. El tamaño del efecto pre-postest fue medio a alto en casi todas las dimensiones de la exploración y medio en la escala de indecisión vocacional.
\end{abstract}

Palabras-clave: proceso; productos; intervención psicológica; vocacional.

\section{CAREer intervention PROCESS AND OUTCOMES}

\begin{abstract}
This study analyzes the process and outcomes of a career counselling intervention. A total of 321 students (178 experimental group y 143 control group), between 13 and 17 years old participated in the study. The counselling process was analysed through the use of the Client Reaction System, while the intervention outcomes assessment included the use of the Career Exploration and Decision Scales. Results of the counselling process analysis evidences that, during the intervention,

^Estudo derivado de dissertação de doutoramento financiada pela Fundação para a Ciência e a Tecnologia (bolsa com a referência FRH/BD/18637/2004).

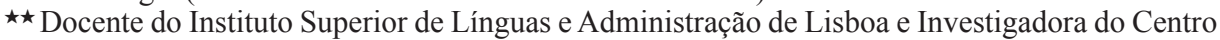
de Investigação em Psicologia, Universidade do Minho - Portugal. Instituto Superior de Línguas e Administração de Lisboa. Quinta do Bom-Nome. Estrada da Correia, No 53, 1500210 Lisboa - Portugal.

$\star \star \star$ Docente da Escola de Psicologia da Universidade do Minho - Portugal e coordenadora do Doutoramento na área de conhecimento em Psicologia Vocacional na mesma Universidade, Universidade do Minho. Escola de Psicologia. Campus de Gualtar. 4710-057. Braga - Portugal. E-mail: ceuta@iep.uminho.pt
\end{abstract}


the reactions of hopeful, relief, better self-understanding, clear, responsibility, new perspective, educated, challenged, and no reaction, registered significant changes in a positive sense throughout the sessions, being the outcome favourable to the intervention. Experimental group had significantly higher scores in several career exploration dimensions and significantly lower scores in career indecision scale. The size of the effect pre-postest was average to high in almost all the career exploration scales, and average in the indecision measure.

Keywords: process; outcomes; career intervention.

\section{INTRODUCCIÓN}

La investigación del proceso de intervención psicológica estudia la forma como el cambio terapéutico si procesa, a través de la identificación de los padrones de comportamiento e interacción del cliente y del psicólogo. La investigación de los productos de la intervención se centra en la evaluación de los cambios en el cliente en el final del proceso, a través de la confrontación con su funcionamiento al principio del mismo (MOREIRA; GONÇALVES; BEUTLER, 2005).

La investigación referente al proceso de la consulta psicológica vocacional evidencia la su gran complexidad (cf. FARIA, 2008), y ofrece una diversidad de metodologías del estudio (e.g., HILL; CORBETT, 1993). Un tipo de estudios del análisis del proceso de la consulta psicológica vocacional es la pesquisa del efecto de las intenciones del psicólogo en las reacciones del cliente (KIRSCHNER; HOFFMAN; HILL, 1994). El análisis indica la existencia de $75 \%$ de acuerdo entre las intenciones del psicólogo y las reacciones del cliente. Los análisis de las medidas del proceso, habían indicado que las intenciones del psicólogo más frecuentes son el cambio, el insight, los sentimientos y el fornecer de información. Por su vez, las reacciones más frecuentes del cliente son el apoyo, el desafío y, la comprensión (KIRSCHNER; HOFFMAN; HILL, 1994). Kirschner, Hoffman y Hill (1994) refieren que los factores responsables para el éxito de la intervención son: la ayuda social, el ofrecimiento de información, la investigación cognitiva de las aspiraciones y la construcción de la comprensión personal y del trabajo.

La investigación meta-analítica referente a los productos de intervención tiene demostrado que la intervención vocacional es moderadamente eficaz con una variedad de clientes (e.g., BROWN; KRANE, 2000; OLIVER; SPOKANE, 1988; SPOKANE; OLIVER, 1983; WHISTON; BRECHEISEN; STEPHENS, 2003; WHISTON; SEXTON; LASOFF, 1998), con una magnitud del efecto entre 0,32 y 0,85 . En lo que respeta a las modalidades de la intervención, se verifica que, la educación para la carrera con los grupos de alumnos (OLIVER; SPOKANE, 1988), la consulta psicológica vocacional individual (WHISTON; SEXTON; LASOFF, 1998), y en grupo (BROWN; KRANE, 2000; SPOKANE; OLIVER, 1983) tiene sido considerabas como las modalidades de la inter- 
vención más eficaces. Por su vez, la consulta psicológica vocacional produce productos más marcados de qué lecciones de información vocacional, y de qué programas asistidos por la computadora (cf. SPOKANE, 2004). Sin embargo, es más o menos consensual que las intervenciones vocacionales donde está ausente la figura del psicólogo, son las que llevan a productos más modestos de los cambios del comportamiento de los clientes.

Tal como en la psicoterapia (e.g., CORBELLA; BOTELLA, 2003; ORLINSKY; HOWARD, 1986; SOARES, 2007), en la consulta psicológica vocacional, la calidad de la relación terapéutica surge asociada a los productos positivos de la intervención (e.g., GURMAN; RAZIN, 1977; HEPPNER, M.; HEPPNER, P., 2003; WHISTON; RAHARDJA, 2008). Con efecto, ja en 1982, Morgan, Luborsky, Cris-Chrisph, Curtis, y Solomon habían demostrado que la correlación entre el grado de la relación terapéutica y los productos positivos de la intervención estaba de 0,50, contribuyendo para $25 \%$ de la variación de los productos. Morgan y colaboradores (1982) habían avanzado, aún, con la idea de que, caso existiera una relación directa causa-efecto entre la relación terapéutica y los productos de la intervención, toda la variabilidad de las medidas de los productos se podría explicar por el grado de relación terapéutica que fue desarrollada. Greenberg y Webster (1982) habían corroborado los descubrimientos de Morgan y colaboradores (1982), avanzando con la hipótesis de que 30\% el 46\% de la variación de los productos en la intervención, pueden ser atribuidos al grado de percepción del cliente acerca del establecimiento de una relación terapéutica en las segundas o terceras sesiones. Sin embargo, la escasa investigación acerca de la relación entre el proceso y los productos de la intervención (BLUSTEIN; SPENGLER, 1995), así como las discrepancias registradas nos estudios de metaanalice, continúan a apelar para la necesidad de si realizar más estudios analíticos referentes a los componentes y procesos que aumentan la eficacia de las intervenciones vocacionales (FARIA, 2008). En esa dirección, el objetivo fundamental de esta investigación es analizar el proceso y los productos de un programa de intervención psicológica vocacional.

\section{MÉTodo}

\section{Participantes}

El programa de intervención fue llevado a cabo de enero a mayo de 2005, con 321 estudiantes de $4^{\circ}$ de la Eso, de ambos sexos (40,8\% varones; 59,2\% mujeres), de cinco escuelas del norte de Portugal, que habían solicitado a la Consulta Psicológica Vocacional de la Universidad del Minho, apoyo específico en la toma de decisión vocacional de sus alumnos. Los jóvenes han sido distribuidos de modo aleatorio en el grupo de intervención y en el grupo control. El grupo experimental estuvo compuesto por 178 estudiantes (58 varones; 120 mujeres) y el grupo control por 143 estudiantes ( 73 varones; 70 mujeres). En ambos los grupos, la edad de los participantes oscila entre 13 y 17 años $(M=15,15 ; S D=0,49)$. 


\section{Variables e instrumentos}

Para evaluar la calidad de la relación terapéutica se utilizó el Sistema de Reacciones de los Clientes - CRS (HILL et al., 1988; adapt. TAVEIRA; OLIVEIRA; GOMES, 2004), un referente al criterio que evalúa 21 reacciones, 14 de las cuales de tonalidad positiva y 7 de tonalidad negativa. Las primeras incluyen las reacciones de comprensión, apoyo, confianza, bienestar, pensamientos/comportamientos negativos, auto-concepto, clareza, reconocimiento de sentimientos, responsabilidad, envolvimiento, alteración de perspectivas, educación, alteración de comportamientos y, mudanza. Las segundas incluyen las reacciones negativas de medo, agravamiento de la situación, desorientación, confusión, incomprensión, bloqueo, y falta de reacción. Cada alumno apunta, para cada una de las sesiones de intervención, la presencia de cada una de las reacciones. Por ejemplo, para a reacción comprensión: "Sentí que el psicólogo realmente me comprendió y percibió lo que yo dice y lo que se pasa conmigo", el alumno coloca un cruce, se corresponde al que se aconteció consigo en la sesión.

Para evaluar los productos de la intervención se han tenido en cuenta las variables exploración e indecisión vocacional. En el primer caso, se utilizó la Career Exploration Survey - CES (STUMPF; COLARELLI; HARTMAN, 1983; adapt. TAVEIRA, 1997). Permite evaluar aspectos cognitivos, comportamentales y afectivos de la exploración de la carrera. La versión original del CES, con un total de 62 ítems, presenta valores aceptables de consistencia interna, entre 0,67 y 0,92 (STUMPF; COLARELLI; HARTMAN, 1983). La versión utilizada está constituida por 54 ítems, con respuesta en formato tipo Likert, entre cinco y siete categorías de respuesta (desde "muy poço", "probabilidad muy baja", "tensión mínima" hasta "muchísima", "probabilidad muy alta", y "mucha tensión"). Los resultados del análisis factorial confirmatorio del CES, efectuada por Taveira (1997), garantizan la plausibilidad de un modelo de medida de doce dimensiones de exploración, estructuradas en tres componentes principales: (i) las creencias de exploración - percepción del estatuto del trabajo (PET), certeza en los resultados de la exploración (CRE), valor instrumental de la exploración del medio (VIELM), valor instrumental de la exploración de sí mismo (VIELS), e importancia de conseguir la posición preferida (IPP); (ii) los comportamientos de la exploración - exploración del medio (EM), exploración de sí mismo (ES), carácter sistemático e intencional de la exploración (CDIE), cantidad de información conseguida (CI); y (iii) las reacciones a la exploración vocacional - satisfacción con la información conseguida (SI), tensión anticipada con la exploración (TE), y tensión anticipada con la toma de decisiones (TTD). Fueran excluidas de esto modelo dimensional, las escalas originales de frecuencia, foco e valor instrumental del método de la exploración. La clasificación de cada subescala del CES se obtiene a través de la adición del valor de cada una de las respuestas a los ítems que la componen, variando las puntuaciones mínimas y máximas de una para otra subescala, en consonancia con el número de ítems de la escala de la respuesta en causa. Los valores más altos se relacionan con la existencia de creencias y de reacciones más positivas a la exploración y una mayor activación del proceso exploratorio (TAVEIRA, 1997). Las escalas del CES se mostraran consistentes 
(alfa de Cronbach de 0,63 a 0,86, para la cantidad de información y la tensión anticipada con la exploración) y relativamente estables (con coeficientes de precisión test-retest de ocho semanas, entre 0,25 y $0,59, \mathrm{p}<0,005)$. El CES demostró aún validad discriminante con relación a las medidas de indecisión e identidad vocacional (TAVEIRA, 1997). En el según caso, se utilizó la Career Decision Scale-CDS (OSIPOW et al., 1976; adapt. TAVEIRA, 1997), composta por 12 ítems, que evalúan el nivel de indecisión vocacional. Los ítems tienen una escala de respuesta tipo Likert, con cuatro categorías, desde 1 ("nada similar a mí") hasta 5 ("exactamente como yo"), en un formato gráfico-numérico en cada una de las alternativas de respuesta. Se pide a los participantes que señalen la categoría que describe con mayor precisión su situación con respecto a cada ítem. Los resultados se consiguen a través de una nota global de la escala para medir la indecisión vocacional. Taveira (1997) demostró la unidimensionalidad y consistencia interna de la CDS (alfa de Cronbach $=0,90$ ).

\section{Programa de INTERVENCIÓN Y PROCEDimiento}

El programa de intervención, titulado "Futuro Bué!", se basa en el modelo de consulta psicológica desarrollista centrado en la relación de Taveira (2001), con cuatro fases: Iniciar, Explorar, Comprometer y Finalizar, cada una de las cuales implica el proseguimiento de objetivos y técnicas específicas. La estructura y los objetivos y técnicas de la intervención se presentan en la tabla 1. Antes de la intervención fue efectuada, una sesión colectiva de divulgación y registro en el programa, y un pretest con los alumnos. En el final de la intervención, fue realizada una sesión postest, semejante a de pretest. La administración de los instrumentos fue colectiva, teniendo una duración aproximada de 45 minutos. Fueran presentados a los alumnos los objetivos del estudio y el interés en el uso de los instrumentos de la investigación, así como se ofrecieron aclaraciones, tales como la confidencialidad de las respuestas dadas.

Se realizaron análisis de estadística descriptiva de los datos de caracterización socio-demográfica de los participantes y de las respuestas globales a las escalas de la exploración e indecisión vocacional. Utilizamos la prueba Q de Cochran para analizar la significación del cambio de las respuestas de los adolescentes en las cinco sesiones de la intervención. Los resultados de los análisis comparativos ínter grupos se consiguieron por medio de la realización de pruebas $t$-Student y Chi-cuadrado. La prueba de hipótesis se basó en los análisis de pruebas de medidas repetidas. Fue, igualmente, efectuada un análisis más detallada del efecto del interacción entre el factor intervención (intervención-control: íntersujeto) y el factor momento de evaluación (pré-pósteste: intrasujeto), se utilizando la prueba multivariada de Pillai. Cuando los análisis divulgan este efecto de interacción se realizó pruebas de test de medidas emparrilladas para determinar el significado de esta interacción. 


\section{Tabla 1 - Estructura general del programa de intervención psicológica vocacional}

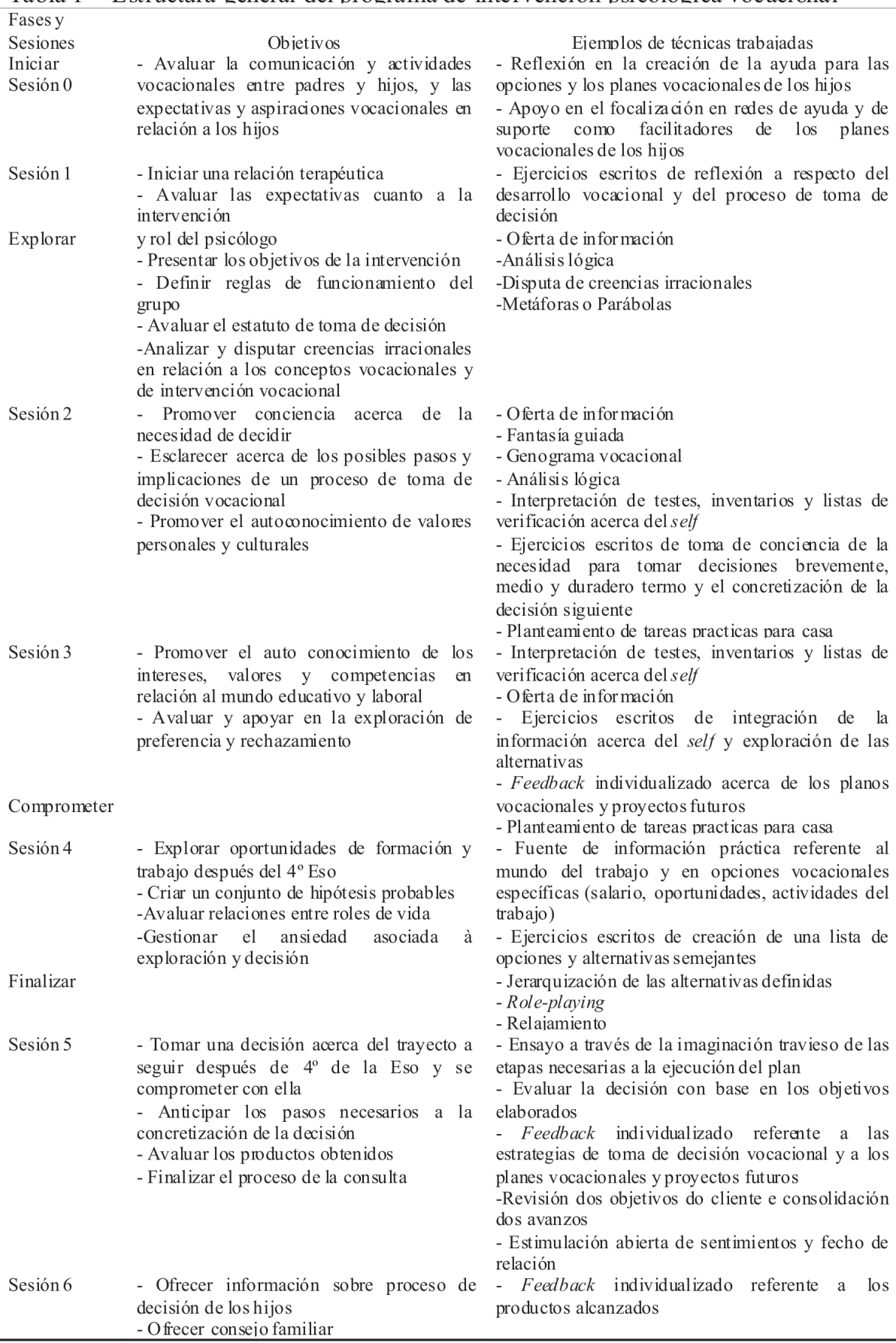




\section{Resultados}

En lo concerniente al análisis del proceso de consulta, se verifica que las reacciones positivas de los clientes predominan sobre las reacciones negativas. La comprensión, la ayuda, la claridad, el bienestar y la confianza son las reacciones positivas más frecuentes a través de todo el proceso de intervención. La confusión, la falta de reacción, el miedo y la desorientación son las reacciones negativas más frecuentes (tabla 2). No total, solamente 9 de las 21 reacciones posibles registraran cambios estadísticos significativos a través del proceso de ayuda relacional, a favor de la intervención.

Tabla 2 - Diferencias en el cociente de reacciones al proceso de la consulta psicológica vocacional: estadísticas descriptivas y nivel de significación

\begin{tabular}{|c|c|c|c|c|c|c|c|}
\hline & $\begin{array}{c}\text { Sesión } \\
1 \\
\text { Frea. }\end{array}$ & $\begin{array}{c}\text { Sesión } \\
2 \\
\text { Frea. }\end{array}$ & $\begin{array}{c}\text { Sesión } \\
3 \\
\text { Frea. }\end{array}$ & $\begin{array}{c}\text { Sesión } \\
4 \\
\text { Frea. }\end{array}$ & $\begin{array}{c}\text { Sesión } \\
5 \\
\text { Frea. }\end{array}$ & $\mathrm{O}$ & $\mathrm{p}$ \\
\hline Comprensión & 168 & 167 & 172 & 161 & 169 & 5.573 & 0.233 \\
\hline Apovo & 138 & 146 & 152 & 146 & 151 & 6.624 & 0.157 \\
\hline Confianza & 101 & 113 & 142 & 128 & 138 & 42.480 & $<0.001$ \\
\hline Bienestar & 112 & 123 & 133 & 130 & 134 & 13.275 & 0.010 \\
\hline Pensamientos Negativos & 51 & 61 & 55 & 50 & 50 & 3.641 & 0.457 \\
\hline Auto concepto & 63 & 95 & 98 & 94 & 101 & 31.101 & $<0.001$ \\
\hline Clareza & 129 & 132 & 136 & 139 & 149 & 9.636 & 0.047 \\
\hline $\begin{array}{l}\text { Reconocimiento de } \\
\text { Sentimientos }\end{array}$ & 77 & 94 & 95 & 92 & 91 & 6.885 & 0.142 \\
\hline Responsabilidad & 79 & 85 & 91 & 108 & 113 & 26.774 & $<0.001$ \\
\hline Envolvimi ento & 92 & 86 & 97 & 100 & 98 & 4.226 & 0.376 \\
\hline $\begin{array}{l}\text { Alteración de } \\
\text { Perspectivas }\end{array}$ & 39 & 60 & 57 & 53 & 55 & 10.852 & 0.028 \\
\hline Educación & 97 & 117 & 92 & 95 & 111 & 14.975 & 0.005 \\
\hline $\begin{array}{l}\text { Alteración de } \\
\text { Comportamientos }\end{array}$ & 24 & 29 & 34 & 36 & 42 & 9.447 & 0.051 \\
\hline Mudanza & 50 & 54 & 53 & 37 & 31 & 18.143 & 0.001 \\
\hline Medo & 6 & 4 & 4 & 8 & 9 & 4.160 & 0.385 \\
\hline $\begin{array}{l}\text { Agravamiento de la } \\
\text { Situación }\end{array}$ & 1 & 4 & 6 & 0 & 4 & 8.571 & 0.073 \\
\hline Bloqueo & 2 & 2 & 2 & 5 & 5 & 3.724 & 0.445 \\
\hline Desorientación & 7 & 7 & 2 & 5 & 3 & 5.073 & 0.280 \\
\hline Confusión & 14 & 11 & 7 & 8 & 5 & 7.143 & 0.129 \\
\hline Incomprensión & 2 & 1 & 1 & 2 & 1 & 0.857 & 0.931 \\
\hline Falta de reacción & 14 & 8 & 4 & 4 & 2 & 17.208 & 0.002 \\
\hline
\end{tabular}

Antes de proceder a la prueba de las hipótesis de los productos de la intervención, analizamos la equivalencia de los grupos, en termos de variables demográficas y psicológicas. Los productos indican que los grupos experimental y control son equivalentes cuanto a la edad, residencia y a la tutela de la escuela, y no equivalentes cuanto al sexo, con más niñas en el grupo experimental $(\chi 2=11,191 ; p \leq 0,01), y$ cuanto a las dimensiones de EM $(t=-2,575 ; p \leq 0,05), C I$ 
$(t=-4,529 ; p \leq 0,01)$, SI $(t=-3,538 ; p \leq 0,01)$, $y$ TTD $(t=2,849 ; p \leq 0,05)$ de la exploración vocacional, así cuánto a la indecisión vocacional $(\mathrm{t}=2,831 ; \mathrm{p} \leq 0,05)$. Los alumnos del grupo experimental, registran, en el pretest, haber explorado poco el mundo profesional; poseer poca cantidad de información apropiada sobre las profesiones, trabajos, organizaciones y sobre sí mismo; poca satisfacción con la información profesional; mayor tensión en la toma de decisión; y niveles superiores de indecisión vocacional en comparación con los del grupo control.

Tabla 3 - Medias y desviaciones típica correspondientes a las variables dependientes en el pre y postest

\begin{tabular}{lcccccccc}
\hline & \multicolumn{3}{c}{ Grupo experimental } & \multicolumn{4}{c}{ Grupo control } \\
& \multicolumn{2}{c}{ Pretest } & \multicolumn{2}{c}{ Postest } & \multicolumn{2}{c}{ Pretest } & \multicolumn{2}{c}{ Postest } \\
\cline { 2 - 9 } PET & M & SD & M & SD & M & SD & M & SD \\
CRE & 9.413 & 2.271 & 10.484 & 1.983 & 9.878 & 2.147 & 10.043 & 2.319 \\
VIELM & 3.205 & 2.908 & 9.156 & 2.679 & 8.533 & 2.917 & 9.411 & 2.807 \\
VIELSM & 14.501 & 6.449 & 38.793 & 6.357 & 37.281 & 7.567 & 37.545 & 6.769 \\
IPP & 10.760 & 2.463 & 11.069 & 2.342 & 10.911 & 2.623 & 10.985 & 2.540 \\
EM & 10.128 & 3.430 & 13.024 & 2.589 & 11.153 & 3.686 & 12.386 & 3.613 \\
ES & 15.497 & 3.982 & 17.061 & 3.856 & 15.745 & 4.076 & 16.223 & 4.089 \\
CSIE & 4.030 & 1.617 & 5.009 & 1.733 & 4.277 & 1.661 & 4.980 & 1.802 \\
CI & 7.205 & 2.351 & 10.070 & 2.386 & 8.427 & 2.465 & 9.656 & 2.339 \\
SI & 8.350 & 2.341 & 10.119 & 2.150 & 9.285 & 2.366 & 9.876 & 2.328 \\
TE & 15.313 & 4.643 & 15.960 & 4.693 & 14.695 & 4.467 & 15.365 & 4.392 \\
TTD & 23.947 & 7.101 & 22.337 & 7.225 & 21.608 & 7.475 & 21.766 & 7.424 \\
IV & 35.966 & 6.132 & 33.006 & 6.876 & 33.895 & 6.962 & 33.531 & 7.420 \\
\hline
\end{tabular}

La tabla 3 presenta los valores del pretest y postest en las dimensiones de exploración e indecisión vocacional para los grupos experimental y control. Los productos de la prueba de hipótesis divulgan la existencia de diferencias estadísticamente significativas entre los momentos pretest y postest, en los grupos experimental y control, para las dimensiones $\operatorname{PET}(\mathrm{F}=13,374 ; \mathrm{p} \leq 0,01), \mathrm{EM}(\mathrm{F}=14,682$; $\mathrm{p} \leq 0,01)$, ES $(\mathrm{F}=6,409 ; \mathrm{p} \leq 0,05)$, SI $(\mathrm{F}=19,580 ; \mathrm{p} \leq 0,01)$, TTD $(\mathrm{F}=3,811 ; \mathrm{p} \leq 0,05)$, e IV $(\mathrm{F}=13,238 ; \mathrm{p} \leq 0,01)$. Las pruebas $t$ demuestran, en el grupo experimental, un aumento estadísticamente significativo para los valores de PET $(\mathrm{t}=-6,182$; $\mathrm{p} \leq 0,01), \mathrm{EM}(\mathrm{t}=-9,981 ; \mathrm{p} \leq 0,01), \mathrm{ES}(\mathrm{t}=6,463 ; \mathrm{p}<0,05), \mathrm{CI}(\mathrm{t}=-5,166 ; \mathrm{p} \leq 0,01)$, SI $(t=-5,573 ; p \leq 0,01)$, e IV $(t=5,921 ; p \leq 0,05)$. En el grupo control apenas se verificaran aumentos estadísticamente significativos para los valores de $\mathrm{EM}(\mathrm{t}=-3,825$; $\mathrm{p} \leq 0,05)$ y de $\mathrm{SI}(\mathrm{t}=-3,156 ; \mathrm{p} \leq 0,05)$. Para evaluar la eficacia de la intervención se calcularan las magnitudes del efecto del programa en cada dimensión psicológica. Seguida la recomendación de Cohen (1977), la magnitud del efecto puedo ser considerada elevada $(\mathrm{d}>80)$ en la EM $(\mathrm{d}=1,21)$, CSIE $(\mathrm{d}=0,95)$ y VIELM $(\mathrm{d}=0,86)$; alta $(50>d<80)$ en la SI $(d=0,79), \operatorname{ELSM}(d=0,58) ;$ media $(20>d<50)$ en la PET $(d=0,50), \operatorname{IV}(d=-0,45)$, CI $(d=0,40), \operatorname{IPP}(d=0,39), \operatorname{CRE}(d=0,35)$, y TTD $(d=-0,22)$; y baja $(d<20)$ en la TE $(d=0,14)$ e, $\operatorname{VIE~} \operatorname{LM}(d=0,10)$. 


\section{Discusión y Conclusiones}

Los productos registrados demuestran que los jóvenes estudiados son competentes en el establecimiento de una relación terapéutica adecuada, y desarrollaran una representación positiva de esta misma. Tenido en cuenta que las reacciones de comprensión, ayuda, claridad, bienestar parecen ser buenos preeditores del establecimiento de una buena alianza terapéutica (e.g., MACHADO; HORVATH, 1999; CONSTANTINO; CASTONGUAY; SCHUT, 2002), y del mantenimiento de los clientes en la consulta (SOARES, 2007), los productos conseguidos son un buen indicador de la evolución y el éxito del proceso de intervención evaluado. El análisis de las reacciones, en los cinco momentos terapéuticos, evidencian también una consistencia entre el sistema de reacciones cognitivas del cliente de Hill y colaboradores (1988) y de las reacciones del modelo de la consulta de Taveira (2001). Sin embargo, aunque los productos, acuerde notar que si debe tener cuidado en su interpretación, dado no haber sido realizado ningún estudio cuantitativo del análisis factorial. Se sugiere, que nuevos estudios de las reacciones de los clientes al proceso de la intervención continúen esta tarea, contribuyendo para el desarrollo del modelo de la consulta y del programa de intervención en causa. Los productos de la comparación pretest-postest en los grupos experimental y control demuestran la eficacia del programa, para la exploración e indecisión vocacional. En concreto, los estudiantes del grupo experimental, después del programa, mejoran significativamente el estatuto del trabajo, exploración con en el medio y en sí mismo, y la satisfacción con la información, y disminuyen la tensión en la toma de decisión y la indecisión vocacional. En el grupo control, este cambio solamente si registró estadísticamente significativo para las dimensiones del estatuto del trabajo y de la satisfacción con la información, lo cual es indicativo de que ésta sería la pauta que seguiría también el grupo experimental si no se hubiera introducido la intervención. Los productos positivos alcanzados por el grupo experimental pueden deber-se à presencia de componentes críticos da eficacia de la intervención vocacional, en lo programa, como la estructura cognitiva para la comprensión personal y del mundo del trabajo, la información vocacional, las narrativas cognitivas y la ayuda social del psicólogo (HOLLAND; MAGOON; SPOKANE, 1981). Así como, tres otros componentes específicos referenciados por Brown y Krane (2000) como responsables del impacto positivo de la magnitud del efecto de la intervención: los ejercicios escritos, el feedback individualizado y la oferta de información actualizada referente al mundo educativo y profesional. Para las dimensiones de la exploración del medio e intencional-sistemática, la magnitud del efecto para el grupo experimental contra el grupo control fue 1,21 y 0,95 , respectivamente. Un producto muy satisfactorio, en comparación con los conseguidos en estudios congéneres anteriores (BROWN; KRANE, 2000; OLIVER; SPOKANE, 1988; SPOKANE; OLIVER, 1983; WHISTON; SEXTON; LASOFF, 1998; WHISTON; BRECHEISEN; STEPHENS, 2003). A excepción de las dimensiones de la tensión en la exploración y valor instrumental interna, todas las dimensiones restantes de la exploración vocacional, y la indecisión vocacional habían presentado magnitudes de efecto medias, según la clasificación de Cohen (1997). Así, en futuras investigaciones del programa, asciende la necesidad de 
llevar en consideración el efecto de la interacción entre el proceso y el producto, de forma a poder prever de una manera más rigurosa, cuales son los factores específicos que permiten explicar los beneficios conseguidos para los jóvenes en el programa de intervención vocacional evaluado.

\section{REFERENCIAS}

BLUSTEIN, D. L.; SPENGLER, P. M. Personal Adjustment: career counseling and psychotherapy. In: WALSH, W.; OSIPOW, S. (Ed.). Handbook of Vocational Psychology: theory, research, and practice. 2nd. ed. Mahwah, NJ: L. Erlbaum, 1995. p. 295-328.

BROWN, S. D.; KRANE, N. E. Four (or five) sessions and a cloud of dust: old assumptions and new observations about career counseling. In: BROWN, S. D.; LENT, R. W. (Ed.). Handbook of Counseling Psychology. 3ed. New York: John Wiley y Sons, 2000. p. 740-749.

COHEN, J. Statistical power analysis for the behavioral sciences. 2th ed. New York: Academic, 1977.

CONSTANTINO, M. J.; CASTONGUAY, L. G.; SCHUT, A. J. The working alliance: a flagship for the "scientist-practioner" model in psychotherapy. In: TRYON, G. S. (Ed.). Counseling based on process research: applying what we know. Boston: Allyn y Bacon, 2002. p. 81-131.

CORBELLA, S.; BOTELLA, L. La alianza terapéutica: historia, investigación y evaluación. Anales de Psicologia, [S.1.], v. 19, n. 2, p. 205-221, 2003.

FARIA, L. A eficácia da consulta psicológica vocacional de jovens: estudo do impacto de uma intervenção. 2008. Tese (Doutorado)-Universidade do Minho, Braga, 2008.

GREENBERG, L. S.; WEBSTER, M. C. Resolving decisional conflict by Gestalt two-chair dialogue: relating process to outcome. Journal of Counseling Psychology, [S.1.], v. 29, n. 5, p. 468-477, Sept. 1982.

GURMAN,A. S.; RAZIN, A. M. Effective psychotherapy: a handbook of research. New York: Pergamon, 1977.

HEPPNER, M. J.; HEPPNER, P. P. Identifying process variables in career counseling: a research agenda. Journal of Vocational Behavior, v. 62, n. 3, p. 429-452, June. 2003. 
HILL, C. E.; CORBETT, M. A perspective on the history of process and outcome research in counseling psychology. Journal of Counseling Psychology, v. 40, n. 1, p. 3-24., Jan. 1993.

HILL, C. E. et al. Development of a system for categorizing client reactions to therapist interventions. Journal of Counseling Psychology, v. 35, n. 1, p. 27-36, Jan. 1988.

HOLLAND, J. L.; MAGOON, T. M.; SPOKANE, A. R. Counseling Psychology: career interventions, research, and theory. Annual Review of Psychology, v. 32, p. $279-305,1981$

KIRSCHNER, T.; HOFFMAN, M.; HILL, C. Case study of the process and outcomes of career counseling. Journal of Counseling Psychology, [S.1.], v. 41, n. 2, p. 216-226, 1994.

MACHADO, P. P.; HORVATH, A. O. Inventário da Aliança Terapêutica: versão portuguesa do Working Alliance Inventory. In: SIMÕES, M. R.; ALMEIDA, L. S.; GONÇALVES, M. (Ed.). Provas Psicológicas em Portugal. Braga: SHO, 1999. v. 2, p. 96-109.

MOREIRA, P.; GONÇALVES, O.; BEUTLER, L. E. Métodos de selecção de tratamento: o melhor para cada paciente. Porto: Porto, 2005.

MORGAN, R. et al. Predicting outcomes of psychotherapy by the Penn Helping Alliance Rating Method. Archives of General Psychiatry, [S.1.], v. 39, n. 4, p. 397-402, Apr. 1982.

OLIVER, L. W.; SPOKANE, A. R. Career-intervention outcome what contributes to client gain? Journal of Counseling Psychology, [S.1.], v. 35, n. 4, p. 447-462, 1988.

ORLINSKY, D. E.; HOWARD, K. I. Process and outcome in psychotherapy. In: GARFIELD, S. L.; BERGIN, A. E. (Org.). Handbook of psychotherapy and behavior change. 3. ed. New York: Wiley, 1986. p.311-384.

OSIPOW, S. H. et al. The Career Decision Scale. Columbus, OH: Marathon; Odessa, FL: Psychological Assessment Resources, 1976.

SOARES, M. L. Parar, pensar e avaliar a psicoterapia - contribuições da investigação de díades de terapeutas e clientes portugueses. 2007. Tese (Doutorado)-Universitat Ramon Llull, Barcelona, 2007. 
SPOKANE, A. R. Avaliação das Intervenções de Carreira. In: LEITÃO, L. M. (Ed.). Avaliação psicológica em orientação escolar e profissional. Coimbra: Quarteto, 2004. p.455-473.

SPOKANE, A. R.; OLIVER, L. W. The outcomes of vocational intervention. In: WALSH; OSIPOW, S. H. (Ed.). Handbook of vocational psychology. Hillsdale, NJ: L. Erlbaum, 1983. p. 99-126.

STUMPF, S. A.; COLARELli, S. M.; HARTMAN, K. Development of the career exploration survey (CES). Journal of Vocational Behavior, [S.1.], v. 22, n. 2, p. 191-226, Apr. 1983.

TAVEIRA, M. C. Exploração e desenvolvimento vocacional de jovens: estudo sobre as relações entre a exploração, a identidade e a indecisão vocacional. 1997. Tese (Doutorado)-Universidade do Minho, Braga, 1997.

TAVEIRA, M. C.O Modelo de intervenção vocacional por programas. Lisboa: Casa Pia de Lisboa, 2001.

TAVEIRA, M. C.; OLIVEIRA, H.; GOMES, D. Sistema de Reacções do Cliente. Braga: Universidade do Minho, 2004. Manuscrito não publicado.

WHISTON, S. C.; BRECHEISEN, B. K.; STEPHENS, J. Does treatment modality affect career counseling effectiveness? Journal of Vocational Behavior, [S.1.], v. 62, n. 3, p. 390-410, June 2003.

WHISTON, S. C.; RAHARDJA, D. Vocational counseling process and outcome. In: BROWN, S. D.; LENT, R. W. (Ed.). Handbook of Counseling Psychology. 4 ed. New York: John Wiley y Sons., 2008. p. 444-461.

WHISTON, S. C.; SEXTON, T.; LASOFF, D. Career-intervention outcome: a replication and extension of Oliver and Spokane (1988). Journal of Counseling Psychology, [S.1.], v. 45, n. 2, p. 150-165, 1998.

Recebido em: 24 de março de 2010

Aceito em: 28 de agosto de 2011 